\title{
Abortion Incidence and Postabortion Care in Rwanda
}

\author{
Paulin Basinga, Ann M. Moore, Susheela D. Singh, Elizabeth E. Carlin, \\ Francine Birungi, and Fidele Ngabo
}

\begin{abstract}
Abortion is illegal in Rwanda except when necessary to protect a woman's physical health or to save her life. Many women in Rwanda obtain unsafe abortions, and some experience health complications as a result. To estimate the incidence of induced abortion, we conducted a national sample survey of health facilities that provide postabortion care and a purposive sample survey of key informants knowledgeable about abortion conditions. We found that more than 16,700 women received care for complications resulting from induced abortion in Rwanda in 2009, or 7 per 1,000 women aged 15-44. Approximately 40 percent of abortions are estimated to lead to complications requiring treatment, but about a third of those who experienced a complication did not obtain treatment. Nationally, the estimated induced abortion rate is 25 abortions per 1,000 women aged 15-44, or approximately 60,000 abortions annually. An urgent need exists in Rwanda to address unmet need for contraception, to strengthen family planning services, to broaden access to legal abortion, and to improve postabortion care. (STUdies IN FAMILy PlanNing 2012; 43[1]: 11-20).
\end{abstract}

Only three years remain to reach the Millennium Development Goal targets. In Rwanda, as in most developing countries, the rate of decrease in maternal mortality is much slower than that needed to achieve the fifth Millennium Development Goal-a 75 percent reduction in the maternal mortality ratio by 2015 (UN 2011). To accelerate progress toward meeting this goal, developing countries need to address the key causes of maternal morbidity and mortality, including unsafe abortion.

The precise level of abortion-related mortality in Rwanda is unknown. The World Health Organization (WHO) estimates that unsafe abortion accounts for one in six maternal deaths in Eastern Africa (WHO 2011). Only slightly more is known about morbidity caused by unsafe abortion. The only published research on this topic - a 2004 study of four health districts in Rwandaestimated that 50 percent of obstetric complications were a consequence of spontaneous and induced abortion

Paulin Basinga is Senior Lecturer, Department of Community Health, and Francine Birungi is Assistant Lecturer, Department of Epidemiology and Biostatistics, School of Public Health, National University of Rwanda. Ann M. Moore is Senior Research Associate and Susheela Singh is Vice President for Research, Guttmacher Institute, 125 Maiden Lane, 7th Floor, New York, NY 10038. Elizabeth E. Carlin is a graduate student, Harvard University, and Fidele Ngabo is Director of Maternal and Child Health Unit, Ministry of Health, Rwanda. E-mail: amoore@guttmacher.org.
(Pearson and Shoo 2005). Abortion's major contribution to maternal morbidity cases that reach health centers strongly suggests that abortion is an important women's health concern in Rwanda and that additional empirical research on this topic is warranted.

\section{Setting and Background}

Abortion law remains restrictive in Rwanda-the procedure is only permitted to save a woman's life or to protect her physical health (Government of Rwanda 1980). Rwanda's abortion law was put into effect in 1977 when the country enacted a new penal code. A woman seeking a legal abortion has to go through the demanding process of obtaining consent for the procedure from two doctors, which makes access to legal abortion extremely difficult even under the limited circumstances when it is allowed under the law. Prison sentences for women who obtain illegal abortions range from one to five years (Kanyesigye 2011), and the penalty for helping a woman to abort outside the legally sanctioned circumstances can be five to ten years (the penalty varies depending on the specifics of the case) (Kwizera 2011). Thus, many medical doctors refuse to provide abortion even when medically indicated (Vyankandondera 2009). Many women turn to illegal abortion to terminate unwanted pregnancies, and some of these procedures are performed by untrained practitioners or under unhygienic conditions and may lead to severe morbidity or death. 
Seventeen years have passed since the 1994 Rwandan genocide, which resulted in a devastating loss of life. A generation of doctors and other health personnel perished and the health infrastructure was nearly destroyed (Ministry of Finance and Economic Planning [Rwanda] 2000). Since that time, Rwanda has made impressive progress in gradually rebuilding its health system and improving access to health services, including reproductive health services (Rusa et al. 2009; Basinga et al. 2011). Between 2005 and 2010, emergency contraception became legal and available, and the percentage of women in union using modern contraceptive methods increased almost five fold, from 9 percent to 44 percent. During the same period, the proportion of births attended by skilled personnel increased by 77 percent, from 39 percent to 69 percent, and under-five mortality declined from 152 to 76 deaths per 1,000 live births (NISR and ORC Macro 2006; NISR, MOH [Rwanda], and ICF International 2011). Improvements in reproductive health care include better availability and quality of maternal health services and the inclusion of postabortion care in the services offered at public health facilities. Uterotonic drugs and antibiotics are included in the national essential drug list and indicated in the national service-delivery guideline for the prevention and treatment of hemorrhage resulting from incomplete abortion. Rwanda's current development plan and poverty-reduction strategy for 2008-2012 place strong emphasis on reproductive health, including family planning (Ministry of Finance and Economic Planning 2007).

In 2010 Rwandan women had an average of 4.6 children, although the number of children they wanted was 3.1 (NISR, MOH [Rwanda], and ICF International 2011). Fifty-two percent of married women were using a contraceptive method in 2010 (NISR, MOH [Rwanda], and ICF International 2011), compared with 17 percent in 2005 (NISR and ORC Macro 2006). Unmet need for family planning declined sharply in recent years: whereas in 2005 unmet need was 38 percent, by 201019 percent of married women did not want a child (or another child) in the next two years or at all and they were not using any method of contraception (NISR, MOH [Rwanda], and ICF International 2011). By 2010, the proportion of Rwandan women who wanted to limit family size or space their next birth was about equal (NISR, MOH [Rwanda], and ICF International 2011). This is a shift from earlier in the decade, when unmet need was predominantly for spacing purposes (NISR and ORC Macro 2006). In addition, many young women become sexually active before marriage, but social sanctions often prevent them from using contraceptives because the practice of contraception among unmarried women is perceived as being an indication of promiscuity. That young women become sexu- ally active a little over half a year prior to marriage, that many are not practicing contraception or not practicing effectively, and that strong sanctions exist against having a child while unmarried are some of the key factors leading young women to obtain abortions (NISR, $\mathrm{MOH}$ [Rwanda], and ICF International 2011; TRAC 2010). Given these conditions, the finding that 43 percent of births in the three years preceding 2008 and 38 percent of births in the five years preceding 2010 were unplanned comes as little surprise (special tabulations of data from the 2007-08 Interim Demographic and Health Survey; NISR, $\mathrm{MOH}$ [Rwanda], and ICF International 2011). ${ }^{1}$ When faced with an unwanted pregnancy, some women will resolve it through abortion.

Data on the overall incidence of induced abortion is a crucial indicator of women's and couples' difficulties in preventing unintended pregnancies and of their need for better contraceptive services. The present study provides the first national and province-level estimates of the incidence of induced abortion and the incidence of treatment for postabortion complications in health facilities in Rwanda's private and public sectors. The study fills an additional gap by providing the first available data on the private sector's provision of postabortion care. Information on the magnitude of unsafe abortion and on its consequences for women's health can be used to help focus public attention on this issue and stimulate government action.

\section{Data and Methods}

The primary data for the study's estimates of abortion incidence were drawn from two surveys conducted by the authors: the Health Facilities Survey and the Health Professionals Survey. Data gathered from these and other sources allowed us to arrive at an estimate of abortion incidence. The study design and protocols were adapted from previous applications of a methodology known as the Abortion Incidence Complications Methodology (AICM) (Singh et al. 2011). We also used data from the 2000, 2005, 2008, and 2010 Rwanda Demographic and Health Surveys. We drew on official national population projections and estimates, and the 2001 and the 2006 Enquête Intégrale sur les Conditions de Vie (EICV) (Integrated Living Conditions Survey) (Ministry of Finance and Economic Planning 2002; NISR 2006 and 2009).

\section{Health Facilities Survey (HFS)}

All health facilities in all sectors (public, public/private [Agrée], ${ }^{2}$ and private) considered to have the capacity to provide postabortion care were included in the sample frame. Health facilities included referral hospitals, dis- 
trict hospitals, private polyclinics (where different types of specialists offer services), private health clinics (which offer general medicine or only one specialty), and public health centers. Health facilities that do not provide primary health-care services or that specialize in nonreproductive services (mental health, tuberculosis, and so forth) and health posts (which lack the capacity to provide postabortion care) were excluded from the sample frame.

The list of health facilities was compiled using data from the Ministry of Health. Because the sample frame received from the Ministry of Health did not contain all private facilities, we worked with the president of the Rwanda Association of Private Medical Doctors to arrive at a comprehensive list of private facilities that potentially provide postabortion-care services. Our sample frame consisted of 466 health facilities, of which 64 percent are public, 29 percent are Agrée, and 8 percent are private. Less than 1 percent of facilities are referral hospitals, 9 percent are district hospitals, less than 1 percent are polyclinics, 7 percent are private health clinics, and 83 percent are public health centers.

We selected 100 percent of hospitals, polyclinics, and private health clinics, and 22 percent of public health centers. The proportion of public health centers selected varied by province, depending on the total number of facilities in each province. We selected a higher proportion of health centers in Kigali (50 percent) because it has a relatively small number of this type of facility, and 19-22 percent in the other provinces (North, South, East, West). This selection process brought our sample frame of 466 facilities down to 167 facilities sampled, of which 165 (99 percent) participated in the survey (see Table 1). Authorization letters from the National Ethics Committee, the National Institute of Statistics, and the Ministry of Health were partly responsible for the nearly universal collaboration of health providers. Another factor contributing to the high response rate was that facility directors were contacted and informed about the visit ahead of time.

Table 1 Sample selection, by type of facility, Health Facilities Survey, Rwanda, 2009

\begin{tabular}{|c|c|c|c|c|c|}
\hline $\begin{array}{l}\text { Type of } \\
\text { health facility }\end{array}$ & $\begin{array}{r}\text { Number } \\
\text { of health } \\
\text { facilities } \\
\text { likely pro- } \\
\text { viding PAC }\end{array}$ & $\begin{array}{c}\text { Percent } \\
\text { sampled }\end{array}$ & $\begin{array}{l}\text { Number } \\
\text { selected }\end{array}$ & $\begin{array}{r}\text { Number } \\
\text { re- } \\
\text { sponded }\end{array}$ & $\begin{array}{r}\text { Re- } \\
\text { sponse } \\
\text { rate } \\
\text { (percent) }\end{array}$ \\
\hline Referral hospital & 3 & 100 & 3 & 3 & 100 \\
\hline District hospital & 41 & 100 & 41 & 41 & 100 \\
\hline Health center & 385 & $22^{a}$ & 86 & 86 & 100 \\
\hline $\begin{array}{l}\text { Private hospital, } \\
\text { polyclinic, clinic }\end{array}$ & 37 & 100 & 37 & 35 & 95 \\
\hline Total & 466 & 36 & 167 & 165 & 99 \\
\hline
\end{tabular}

At each selected facility, a senior staff member knowledgeable about the facility's postabortion-care provision was asked to respond to the survey. In hospitals, the respondent was usually the chief of the obstetrics and gynecology department; in health clinics and health centers, the respondent was typically the facility's director. All potential respondents were read an informed consent statement before survey administration began. No remuneration was offered. The informants were asked whether their facilities provide treatment for abortion complications in an outpatient setting, an inpatient setting, or both. If a respondent indicated that the facility provides treatment, he or she was asked to estimate the number of postabortion patients (spontaneous and induced combined) ${ }^{3}$ treated as outpatients and as inpatients, for two reference periods: an average month and the past month. Specifying two time frames increases the likelihood of accurate recall and of capturing variation from month to month. These two numbers were subsequently averaged and multiplied by 12 to produce an estimate for the calendar year.

\section{Health Facility Survey (HFS) Data Collection}

Fieldwork was carried out from May to August 2010. The HFS was conducted by a field team recruited from a pool of experienced data collectors who had previously worked for the National Institute of Statistics or graduated from either the National University of Rwanda's medical school or from one of Rwanda's nursing schools. The field team participated in a weeklong training session held jointly by staff from the National University of Rwanda School of Public Health and the Guttmacher Institute. All the interviewers were fluent in French, and data collection was carried out in French. Part of the training included pretesting the questionnaires in the field. The field team was organized into four groups of four individuals: three interviewers (nurses or advanced medical students) and one supervisor (a medical doctor, an experienced nurse, or a district health supervisor). ${ }^{4}$

All the field teams started in Kigali, where the incountry principal investigator (PI) and all members of the study team were based. This allowed the PI to closely monitor interviewer performance, implementation of field protocols, and challenges. Once the HFS fieldwork had concluded in Kigali, the field teams covered the remaining provinces.

The HFS data were weighted to project the results nationally, taking into account a facility's probability of selection into the sample, by province and facility type, and the proportion of facilities responding. The weighting factor for a given facility type was the inverse of that subgroup's sampling ratio multiplied by the proportion of completed interviews among sampled facilities. 


\section{Health Professionals Survey (HPS)}

Respondents to this survey are individuals recognized to be highly knowledgeable about abortion provision and postabortion care in Rwanda. Factors considered in selecting respondents included affiliation, expertise in and experience with abortion provision and postabortion care, and reputation among local stakeholders in the field of reproductive health. Drawing upon the input of a number of knowledgeable individuals, including members of the Ministry of Health's Maternal and Child Health section, we compiled a list of key informants.

A purposive sample of 59 key informants from throughout the country was selected; 56 respondents were successfully interviewed, for a response rate of 95 percent. Selected respondents included general medical practitioners, obstetrician/gynecologists, nurses (A1 and A2), ${ }^{5}$ midwives, other health professionals, social workers, a hairdresser, academics, government employees (including parliamentarians), and Rwandans involved in bilateral and multilateral organizations working in this area, from the public and private sector. Respondents were asked about their perceptions of various aspects of induced abortion, such as from whom women obtain abortions, the probability of experiencing complications requiring medical care, and the probability that a woman requiring medical care would receive it. A particular effort was made to have sufficient representation of respondents knowledgeable about the context of abortion in rural areas. Approximately 40 percent of the respondents had worked in rural areas for six months or longer, and almost the same proportion worked primarily in rural areas. Respondents came from all five provinces: Kigali $(n=23)$, South $(n=14)$, West $(n=8)$, East $(\mathrm{n}=7)$ and North $(\mathrm{n}=4)$.

Conducting HPS interviews required greater skill than that required for the HFS interviews, in part because respondents included senior professionals. HPS interviews were conducted by the principal investigator (Paulin Basinga), co-investigators, and field supervisors. Appointments were scheduled for an appropriate interview time, and potential respondents were asked whether they were most comfortable with French or English. Interviewers were matched based on language ability, and all interviewees gave written consent to participate.

\section{Estimating the Incidence of Induced Abortion}

For this study, the total number of women having abortions in a year includes those who were treated for complications at health facilities, those who had complications and either received no care or obtained care from sources other than health facilities (including those who died before obtaining care), and those who had no complications. Following the approach used in previous studies, we estimated the incidence of induced abortion first by estimating the annual number of women receiving treatment for complications from induced abortion at health facilities, and then applying a multiplier that represents the proportion of women having an abortion who do not obtain treatment at health facilities or do not need treatment (Singh et al. 2011).

Because complications of spontaneous and induced abortion are often similar, and because legal restrictions on induced abortion may lead to misreporting of the number treated for complications from induced abortion, correctly categorizing the cause of a pregnancy loss at the facility level is difficult. We therefore relied on an indirect method of estimating the number of women treated for spontaneous abortion. Spontaneous abortions that are likely to require care at a health facility are those that occur in the second trimester, and spontaneous pregnancy loss at 13-21 weeks gestation is estimated to be 3.41 percent of live births, based on life tables from clinical studies of pregnancy loss (Harlap et al. 1980; Bongaarts and Potter 1983). ${ }^{6}$ Applying this percentage to the number of births occurring nationally and in each province, we estimated the number of second-trimester pregnancies ending as spontaneous abortions. The estimated number of births in Rwanda in 2009 was calculated based on agespecific fertility rates from the 2008 DHS and estimates for 2009 of the number of women in each five-year age group, nationally and for each of the five provinces.

Only a proportion of women who need treatment for complications of late spontaneous abortions will have access to a health facility. We relied on the percentage who delivered at a health facility (among all women giving birth) as measured by the DHS as a proxy for the likelihood that a woman with complications from a spontaneous abortion will obtain care at a health facility. The proportion delivering at a health facility in 2009 was estimated for Rwanda and for each province based on the annual rate of increase between the two recent DHS surveys: 2008 and 2010. Based on DHS surveys for those years, a 15 percent annual rate of change was found, and the proportion of women delivering in hospitals was estimated to be 61 percent in 2009. Applying these proportions, we estimated that nationwide in 2009, 8,980 women were treated at health facilities for complications of late spontaneous abortions. Subtracting this number from the total number of women receiving postabortion care in 2009, we estimated that 16,749 women were treated for complications of induced abortion.

Many women who have abortions do not receive postabortion care. Some do not experience complications, and some who have complications do not succeed 
in obtaining care at a health facility. To estimate the total number of women obtaining abortions, we constructed a multiplier to capture the proportion of all women obtaining abortions who are not treated for complications at health facilities. The multiplier was calculated using three figures derived from data from the HPS: the distribution of all women obtaining an abortion according to type of provider, the proportion who experience complications according to type of provider, and the proportion of women with complications who are estimated to obtain care from a health facility. Because conditions under which women obtain abortions likely vary greatly by socioeconomic status and place of residence, these questions were asked separately for the following four subgroups of women: urban poor, urban nonpoor, rural poor, and rural nonpoor. We then calculated the percentage expected to be hospitalized for complications from induced abortion among all women having abortions in each of these four subgroups, for two areas (Kigali and all other provinces), because the HPS sample size was too small to permit calculating multipliers for each province. These percentages were then weighted by the proportional size of the population subgroups to arrive at a multiplier for each of these two areas.

\section{Estimating Unintended Pregnancy}

To calculate numbers and rates of unintended pregnancythe root cause of induced abortion-we first calculated the national and province-specific number of unplanned births. We did so by applying the proportion of births that are unplanned (mistimed or unwanted at the time of conception) according to the 2008 DHS (the most recent national data that are available) to the estimated total number of live births in 2009. Combining this number with the number of induced abortions and including an estimate of the number of miscarriages that resulted from unintended pregnancies yielded an estimate of the total number of unintended pregnancies occurring in Rwanda in $2009 .{ }^{7}$ We then calculated the rate of unintended pregnancies per 1,000 women of reproductive age and the proportion of all pregnancies that were unintended.

\section{Measuring Contraceptive Use}

We obtained the proportion of women using contraceptive methods, both modern and traditional, from the 2005, 2008, and 2010 DHS surveys. Modern methods were defined as pills, injectables, male and female sterilization, IUDs, implants, condoms, and spermicides. Traditional methods included the lactational amenorrhea method (LAM), the standard days method, withdrawal, and folk methods. ${ }^{8}$

\section{Results}

\section{Provision of Postabortion Care}

An estimated 92 percent of all facilities in the country treat postabortion complications (see Table 2). Public facilities must provide a comprehensive package of services for the management of postabortion complications if they are a referral or district hospital, or a minimum package of services if they are a health center. Perhaps surprisingly, all Agrée facilities reported providing postabortion care (not shown). Private facilities were the only type of facility where postabortion care was not universally provided: 22 of the 35 private facilities (63 percent) reported providing postabortion care (1 hospital, 1 polyclinic, and 20 clinics) (not shown). Lower rates of postabortion provision at private facilities may be the result of several factors. First, although the types of services that private clinics are supposed to provide are clearly defined by the Ministry of Health, these results indicate that some private clinics do not comply with regulations. Another possible reason is that investing in the training, equipment, and other commodities needed for provision of postabortion care may be expensive. Private clinics may find it more cost-effective to refer these cases to public facilities.

The average annual caseload of postabortion patients (including spontaneous and induced abortions) treated per facility that offers postabortion care is 57 (Table 2).

Table 2 Indicators of availability of postabortion services provision, by type of facility, according to province and nationally, Rwanda, 2009

\begin{tabular}{|c|c|c|c|c|c|c|}
\hline $\begin{array}{l}\text { Indicators of service } \\
\text { provision and use }\end{array}$ & National & Kigali & North & South & East & West \\
\hline \multicolumn{7}{|l|}{ Availability } \\
\hline $\begin{array}{l}\text { Number of facilities } \\
\text { potentially providing PAC }\end{array}$ & 466 & 64 & 83 & 111 & 100 & 108 \\
\hline Number responded & 165 & 51 & 22 & 32 & 27 & 33 \\
\hline Number providing PAC & 152 & 38 & 22 & 32 & 27 & 33 \\
\hline Percent providing PAC & 92 & 75 & 100 & 100 & 100 & 100 \\
\hline \multicolumn{7}{|l|}{$\begin{array}{l}\text { Annual average PAC case- } \\
\text { load per type of facility }\end{array}$} \\
\hline \multicolumn{7}{|l|}{ Type } \\
\hline Referral hospital & 484 & 396 & - & 660 & - & - \\
\hline District hospital & 200 & 441 & 90 & 176 & 197 & 199 \\
\hline Health center & 37 & 36 & 37 & 28 & 31 & 55 \\
\hline Private facility & 75 & 83 & - & - & - & 2 \\
\hline \multicolumn{7}{|l|}{ Ownership } \\
\hline Public facility & 50 & 120 & 46 & 41 & 37 & 54 \\
\hline Agrée facility & 70 & 51 & 25 & 58 & 104 & 93 \\
\hline Private facility & 75 & 83 & - & - & - & 2 \\
\hline All facilities offering PAC & 57 & 100 & 41 & 47 & 45 & 70 \\
\hline \multicolumn{7}{|l|}{$\begin{array}{l}\text { Complications treated per } \\
1,000 \text { women aged } 15-44\end{array}$} \\
\hline All abortions & 10.7 & 21.5 & 8.2 & 8.1 & 8.3 & 13.3 \\
\hline Induced abortions & 7.0 & 17.8 & 4.7 & 4.7 & 4.3 & 9.3 \\
\hline
\end{tabular}


Referral hospitals, with the largest capacity of all types of facilities, treat an average of 484 postabortion patients per year; district hospitals average 200 such patients per year, and health centers only 37 . Although public facilities care for the majority of women treated for abortion complications (60 percent), Agrée facilities treat 33 percent and private facilities treat 6 percent (not shown). Public health insurance (Mutuelle) increases the likelihood that women will seek care for treatment of postabortion complications at a public facility, because postabortion care is included in the package of services covered.

\section{Abortion Morbidity}

Using data from the HFS, we estimated that 25,727 women received care for complications of spontaneous and induced abortions in Rwandan health facilities in 2009 (see Table 3). We avoided duplication in counts of women treated in more than one facility by asking each facility about the number referred out, and deleting those cases from the overall count for that facility. Nationally, there were 433,697 live births in 2009 in Rwanda and an estimated 14,790 late spontaneous abortions (see Table 3). Subtracting the estimated number of women who were treated in facilities for complications resulting from spontaneous abortion, we estimate that about 16,700 women were treated for complications resulting from induced abortions. Nationally, the rate of hospitalization for treatment of induced abortion complications was 7 per 1,000 women of reproductive age (15-44). Since an estimated 40 percent of all women having induced abortions experienced complications that required medical treatment, 12 percent of all women having induced abortions, or about 30 percent of those who had a complication, did not obtain needed care.
The treatment rate was much higher in Kigali (18 per 1,000 women), somewhat higher in the West province (9 per 1,000 women), and below average in the North, South, and East provinces (rates of 4-5 per 1,000) (see Table 3 ). If all other factors remained the same, and if relatively few women had multiple unsafe abortions for which they required treatment of complications, the overall rate suggests that 21 percent of women ( 7 per 1,000 multiplied by 30 [years from 15-44] $=210$ per 1,000) will require treatment for complications of an induced abortion over their reproductive lifetimes.

According to the key experts surveyed, the majority of abortions among relatively well-off women are likely performed by trained health professionals, such as doctors, medical health assistants, nurses (A1 or A2), or midwives. Among poor women, access to trained health professionals is greater for those living in urban than in rural areas. According to data from the HPS survey, physicians who provide abortions in Rwanda reportedly commonly use dilation and curettage (D\&C) as well as oral and vaginal administration of hormonal medications. Complications may result, however, from procedures conducted by trained providers who have little experience or who work in unhygienic settings. Moreover, a substantial proportion of abortions among all subgroups of women entail a high risk of complications because they are performed by informal and untrained providers (traditional healers, lay practitioners, pharmacists, or women themselves). Informal abortion providers in urban areas are thought to commonly administer hormonal and other types of drugs (taken orally or inserted into the vagina) and to insert herbs or metal objects into the vagina. Informal providers in rural areas and women who induce their own abortions are believed to commonly use decoctions of substances mixed with water or alcohol (admin-

Table 3 Number of women treated for abortion complications, by province and nationally, Rwanda, 2009

\begin{tabular}{|c|c|c|c|c|c|c|c|}
\hline \multirow[b]{2}{*}{ Province } & \multirow[b]{2}{*}{$\begin{array}{c}\text { Women } \\
\text { aged } 15-44^{\mathrm{a}}\end{array}$} & (2) & (3) & \multirow{2}{*}{$\begin{array}{r}(4) \\
\text { Late } \\
\text { miscarriages } \\
\text { among women } \\
\text { aged 15-49 }\end{array}$} & \multirow{2}{*}{$\begin{array}{r}\text { (5) } \\
\text { Women } \\
\text { treated for } \\
\text { miscarriages in } \\
\text { health facilities }\end{array}$} & \multirow{2}{*}{$\begin{array}{l}\text { (6) } \\
\text { Women treated for } \\
\text { induced abortion } \\
\text { complications in } \\
\text { health facilities }^{f}\end{array}$} & \multirow{2}{*}{$\begin{array}{r}(7) \\
\text { Rate of treatment } \\
\text { for induced } \\
\text { abortion } \\
\text { complications }\end{array}$} \\
\hline & & $\begin{array}{l}\text { Women treated } \\
\text { for abortion } \\
\text { complications }^{b}\end{array}$ & $\begin{array}{r}\text { Live births } \\
\text { among women } \\
\text { aged } 15-49^{c}\end{array}$ & & & & \\
\hline Kigali city & 237,172 & 5,096 & 34,102 & 1,163 & 877 & 4,219 & 17.8 \\
\hline North & 415,208 & 3,386 & 72,276 & 2,465 & 1,427 & 1,959 & 4.7 \\
\hline South & 639,025 & 5,173 & 112,076 & 3,822 & 2,196 & 2,978 & 4.7 \\
\hline East & 550,359 & 4,550 & 108,593 & 3,703 & 2,209 & 2,342 & 4.3 \\
\hline West & 565,889 & 7,522 & 106,650 & 3,637 & 2,271 & 5,251 & 9.3 \\
\hline National total & $2,407,652^{h}$ & $25,728^{h}$ & 433,697 & $14,789^{h}$ & 8,980 & $16,748^{\mathrm{h}}$ & 7.0 \\
\hline
\end{tabular}

a Source: National Population Projection 2007-22. $\quad$ ' Includes spontaneous and induced abortions among all women. Source: Health Facilities Survey. ${ }^{\circ}$ Calculated by applying age-specific fertility rates to estimates of the number of women in each five-year age-group. Source: 2008 Rwanda DHS. ${ }^{\mathrm{d}}$ Miscarriages at 13-21 weeks gestation, based on clinical data on miscarriage rates (Harlap et al. 1980; Bongaarts and Potter 1983), calculated as 3.41 percent of all live births among women aged 15-49 (column $3 \times 0.0341$ ). eProportion of women experiencing miscarriages who obtain treatment is presumed to equal the proportion who delivered in health facilities in 2009 (column 4 x province-specific multiplier calculated from the 2008 and 2010 Rwanda DHSs). $\quad$ 'Calculated by subtracting the number of women treated for care of miscarriage from the number treated for any abortion complication (column 5 - column 2). $\quad$ 'Rate per 1,000 women aged $15-44$ [(column $6 \div$ column 1 ) $\times 1000]$. ${ }^{n}$ National total may differ from the sum of individual provinces as a result of rounding.

Note: Following the convention established by WHO (2011), and to maintain comparability with international and other national studies, we present the rate of treatment for induced abortion complications per 1,000 women aged 15-44. The numerator of this rate is slightly disproportionate to the denominator because it is drawn from women aged 15-49, but because few abortions are among women aged $45-49$, a smaller bias is introduced by using the WHO convention for calculating this rate than if we included women aged $15-49$ in the denominator. 
istered vaginally or orally), to insert products into the vagina, and to ingest orally drugs and other substances to induce abortion.

\section{Abortion Incidence}

Table 4 shows our estimates of the number of induced abortions in Rwanda in 2009, which were calculated from the product of a multiplier and the number of women treated for postabortion complications. The multiplier estimated for Kigali was 4.87 and for all other provinces 3.17. The sum of the province-level estimates (essentially equal to a weighted average) yielded the national multiplier of 3.6. Given that our estimate of abortion incidence is an approximation of the true incidence, presenting a range of estimates is appropriate. Low and high estimates were calculated by adding -1 and +1 to the multipliers. The lower the multiplier, the less safe abortion is and / or the more access women have to medical care, whereas the higher the multiplier, the more safe abortion is and/or the less access women have to medical care. Using the national multiplier of 3.6 suggests that an estimated 60,276 induced abortions occurred in Rwanda in 2009. The estimates using the low and the high multipliers ranged from 44,000 to 77,000 .

Nationally, the medium annual abortion rate for 2009 was estimated to be 25 induced abortions per 1,000 women aged 15-44 (see Table 5). The low and high estimates ranged from 18 to 32 per 1,000 women. The abortion rate varied greatly across provinces. Kigali was estimated to have a much higher rate (87 per 1,000 women annually, medium estimate) than the other provinces (range of 14 to 29 per 1,000). This high rate likely reflects, in part, the influx of women from areas outside the city because of the scarcity of both abortion provision and postabortion care elsewhere. The medium estimates for the North, South, and East provinces were in the range of 14 to 15

Table 4 Number of women treated in a health facility for complications of unsafe induced abortion, and estimated total number of induced abortions, by province and nationally, according to multiplier, Rwanda, 2009

\begin{tabular}{lrrrr}
\hline & $\begin{array}{r}\text { Number of women } \\
\text { treated for induced }\end{array}$ & \multicolumn{3}{c}{$\begin{array}{c}\text { Estimated total number of } \\
\text { induced abortions, by multiplier }\end{array}$} \\
\cline { 3 - 5 } & abortion complications & $\mathbf{2 . 6}$ & $\mathbf{3 . 6} \mathbf{6}^{\mathrm{a}}$ & $\mathbf{4 . 6}$ \\
\hline Kigali & 4,219 & 16,341 & 20,560 & 24,778 \\
North & 1,959 & 4,251 & 6,210 & 8,169 \\
South & 2,978 & 6,461 & 9,439 & 12,417 \\
East & 2,342 & 5,081 & 7,422 & 9,764 \\
West & 5,251 & 11,394 & 16,645 & 21,896 \\
National total & 16,749 & 43,528 & 60,276 & 77,024 \\
\hline
\end{tabular}

${ }^{a}$ Different multipliers were calculated for Kigali than for the rest of the country. The multiplier for Kigali was 4.87 and the multiplier for the rest of the country 3.17. A weighted average of province-level estimates yields the national multiplier (3.60). Sources: Estimates based on Health Facilities Survey and Health Professionals Survey.
Table 5 Estimated abortion rate and abortion ratio, by province and nationally, according to level of multiplier, Rwanda, 2009

\begin{tabular}{lrrrrrrrr} 
& \multicolumn{3}{c}{$\begin{array}{c}\text { Abortion rate by } \\
\text { level of multiplier }\end{array}$} & & \multicolumn{3}{c}{$\begin{array}{c}\text { Abortion ratio by } \\
\text { level of multiplier }\end{array}$} \\
\cline { 2 - 4 } Province & Low & Medium & High & & Low & Medium & High \\
\hline Kigali & 68.9 & 86.7 & 104.5 & & 47.9 & 60.3 & 72.7 \\
North & 10.2 & 15.0 & 19.7 & & 5.9 & 8.6 & 11.3 \\
South & 10.1 & 14.8 & 19.4 & & 5.8 & 8.4 & 11.1 \\
East & 9.2 & 13.5 & 17.7 & & 4.7 & 6.8 & 9.0 \\
West & 20.1 & 29.4 & 38.7 & & 10.7 & 15.6 & 20.5 \\
National total & 18.1 & 25.0 & 32.0 & & 10.0 & 13.9 & 17.8 \\
\hline
\end{tabular}

Notes: Abortion rate is the number of induced abortions per year per 1,000 women aged 15-44. Abortion ratio is the number of induced abortions per 100 live births.

Sources: Health Facilities Survey; Health Professionals Survey; National Population Projection 2007-22.

per 1,000, whereas the estimate for the West province was somewhat higher, at 19 per 1,000.

At the national level, an estimated 14 abortions occurred per 100 live births in 2009 (Table 5, medium estimate). The abortion ratio was much higher in Kigali-60 abortions per 100 live births-than in other provinces, where this ratio ranged between 7 and 16. As in the case of the abortion rate, this measure, which was drawn from the indirect methodology estimating postabortion complications treated in facilities, would also be affected by women from other provinces seeking postabortion care in Kigali. Consequently, the abortion ratio is likely overestimated for Kigali and underestimated for other provinces.

\section{Abortion in the Context of Unintended Pregnancies}

Combining our estimates of induced abortion for 2009 with an estimate of the number of unplanned births based on data from the 2008 DHS, we estimated the total number of unintended pregnancies in 2009. The proportion of unplanned births during the three-year period before the 2008 survey was applied to the total number of live births in 2009, assuming that this proportion changed little over this short period.

The results show that 114 unintended pregnancies occurred per 1,000 women in 2009 nationwide, and 47 percent of all pregnancies were unintended (see Table 6). Given the high abortion rate in Kigali, that the province had the highest unintended pregnancy rate (172 per $1,000)$ and the highest proportion of all pregnancies that were unintended (64 percent) among the five provinces was not surprising. The unintended pregnancy rate was close to the national level in three provinces (North, East, and West), with unintended pregnancy rates ranging between 110 and 117 . The South had a notably lower unintended pregnancy rate (93 per 1,000 women), although this is still high in absolute terms. 
Table 6 Number and rate of pregnancies and unintended pregnancies, and number of unplanned births, by region and nationally, Rwanda, 2009

\begin{tabular}{|c|c|c|c|c|c|}
\hline Province & $\begin{array}{r}\text { Number of } \\
\text { preg- } \\
\text { nancies }^{\mathbf{a}}\end{array}$ & $\begin{array}{r}\text { Unintended } \\
\text { pregnancy } \\
\text { rate }^{\mathrm{b}}\end{array}$ & $\begin{array}{r}\text { Percent of } \\
\text { unintended } \\
\text { pregnancies }\end{array}$ & $\begin{array}{l}\text { Preg- } \\
\text { nancy } \\
\text { rate }^{c}\end{array}$ & $\begin{array}{r}\text { Number of } \\
\text { unplanned } \\
\text { births }\end{array}$ \\
\hline Kigali & 63,538 & 172 & 64 & 268 & 15,073 \\
\hline North & 93,562 & 115 & 51 & 225 & 34,259 \\
\hline South & 144,874 & 93 & 41 & 227 & 40,796 \\
\hline East & 138,476 & 117 & 46 & 252 & 46,695 \\
\hline West & 146,289 & 110 & 42 & 259 & 36,474 \\
\hline National total & 586,739 & 114 & 47 & 244 & 173,297 \\
\hline \multicolumn{6}{|c|}{$\begin{array}{l}\text { aPregnancies include births, induced abortions, and miscarriages. } \\
\text { unintended pregnancies per year per } 1,000 \text { women aged } 15-44 . \\
\text { pregnancies per year per } 1,000 \text { women aged } 15-44 \text {. } \\
\text { Notes: Population estimates for } 2009 \text { are based on national population estimates } \\
\text { from the National Population Projection } 2007-22 \text { and the } 2008 \text { Rwanda DHS. Age- } \\
\text { specific fertility rates (ASFRs) obtained from the } 2008 \text { DHS were applied to the } \\
2009 \text { population of women by five-year age groups to estimate number of births in } \\
2009 \text {. Proportion of unplanned births (unwanted or mistimed, also from the } 2008 \\
\text { DHS) was applied to total number of births (nationally and by region) to obtain the } \\
\text { number of unplanned births in } 2009 \text {. The number of abortions and miscarriages } \\
\text { are estimates developed by the authors (see Methods section). }\end{array}$} \\
\hline
\end{tabular}

\section{Factors Underlying Unintended Pregnancy and} Abortion

We examined differences across the five provinces in the use of any contraceptive method, in the use of modern methods, and in fertility preferences to see whether these differences were associated with differences in levels of unintended pregnancy and abortion (not shown). Nationally, the proportion of women in union aged 15-49 who were currently using a modern contraceptive method rose from 4 percent in 2000 to 10 percent in 2005 and then rose sharply to 27 percent in 2008 and 45 percent in 2010 (ONP and ORC Macro 2001; NISR and ORC Macro 2006; $\mathrm{MOH}$ [Rwanda] et al. 2009; NISR, MOH [Rwanda], and ICF International 2011). The proportion using a modern method in Kigali increased from 20 percent to 45 percent over the 2005-10 period. (In the other provinces, the rates climbed from 7-9 percent to 34-35 percent.) Nationally, the proportion using any traditional method changed little over recent years-9 percent in 2000, 8 percent in 2005, and 10 percent in 2008.

According to the DHS, the proportion of births in Rwanda reported as unplanned (unwanted or mistimed) rose from 34 percent in 2000 to 39 percent in 2005, and remained at this high level (38 percent) in 2010 (not shown). Nationally, the gap between the number of children that women want and the number they actually have increased from 1.1 children in 2000 to 1.5 children in 2005 and remained at 1.5 children in 2010, even as average family size fell from 5.8 to 4.6 (not shown). The proportion of women in union in 2010 who had an unmet need for contraception-that is, women who did not want a child within the next two years or wanted no more children but were not using a contraceptive method-rose from 18 percent in 2000 to 38 percent in 2005, and then declined steeply, reaching 19 percent by 2010. The very sharp in- crease in contraceptive use from 2005-10 (from 17 percent to 52 percent using any method among women in union) is consistent with the steep decline in unmet need.

\section{Discussion}

The 2009 rate of abortion in Rwanda-25 abortions annually per 1,000 women aged 15-44-is moderate compared with the 2008 rate for Eastern Africa, which is estimated to have an abortion rate of 36 abortions per 1,000 women aged 15-44, but is similar to the 2008 rate of 28 for Africa as a whole (WHO 2011). The estimated abortion rate in Uganda is 54, in Ethiopia 23, and in Malawi 24 (Singh et al. 2005; Singh et al. 2010; Levandowski et al. 2011). Rwanda's rate puts it on par with a number of countries in the region, and quite a bit below neighboring Uganda.

The higher abortion rate in Kigali, compared with the rest of the country, warrants further discussion. The province-level estimates are subject to greater error than the national estimate, because of unmeasured behavior such as women traveling across provincial boundaries to obtain abortion-related services. Province-specific data, however, identify where women are seeking services, and thus have implications for demand on the health-care system. The high estimate for Kigali, for example, though partly a reflection of cross-provincial travel, is useful in pointing to the great need in this province for services concerning unintended pregnancy and safe abortion.

In addition to cross-provincial travel, several other explanations for Kigali's higher-than-average abortion rate are plausible. Young women in Kigali are more likely to complete higher levels of education and to enter the labor force, increasing the likelihood that an unintended pregnancy might jeopardize their life plans. Additionally, a larger gap (2.0 years) exists between the median age at first intercourse and first marriage in this province, compared with 0.8 years in the South province and 0.4 years in the other three provinces (NISR, MOH [Rwanda], and ICF International 2011). Each of the above factors contributes to the number of unplanned pregnancies in the province. Kigali's higher multiplier also suggests that women in this province are more likely than women in other provinces to obtain safer abortions, in part because they may be able to access services from trained professionals. Another draw that Kigali offers is greater anonymity and confidentiality concerning abortion services than rural areas can provide. Further research is needed to capture place of residence of women obtaining postabortion care.

The estimation methodology employed in this study incorporates inputs from multiple sources to increase the validity of the results. Some of the parameters used in the calculation are global figures (such as the proportion of 
later-term pregnancies that end in miscarriage), assumptions based on DHS estimates (the proportion of women experiencing later-term miscarriages who obtain care in a health facility), or estimates by knowledgeable experts (the number of women treated for postabortion complications in each facility in 2009). A range of estimates is presented (based on assuming a multiplier of +1 and -1 ) as a means of capturing measurement error in the HPS data, and sample error in the HFS count of women treated for postabortion complications. Until reliable data are available to measure empirically the first two of these factors for Rwanda specifically, estimation of abortion incidence in Rwanda will continue to rely on these types of inputs and be subject to the sensitivity of these inputs, given the context of a highly restrictive abortion law.

Unsafe abortion endangers women's health. In Rwanda in 2009, more than 16,700 women received care for complications resulting from induced abortion, and almost one-third of those who experienced postabortion complications did not receive treatment. Despite the health-delivery gains the country has made since the 1994 genocide, some important gaps and needs remain, especially in regard to abortion-related care and prevention of unintended pregnancy. Greater efforts are needed to improve provision of postabortion care. According to our Health Facilities Survey, 85 percent of hospitals report having vacuum aspiration supplies and 97 percent report having a dilation and curettage $(D \& C)$ kit, but only 13 percent of health centers and 7 percent of polyclinics have this equipment. Consistent with this information about equipment, most hospitals (referral and district level) report using surgical methods (evacuation and $D \& C$ ), although even these larger facilities do not commonly use manual vacuum aspiration (MVA). In contrast, most health centers use manual curettage for treating postabortion patients; few use MVA or any surgical procedures. In addition, misoprostol is still unavailable in most of Rwanda. Most providers, therefore, do not employ the techniques recommended by WHO for treating uncomplicated postabortion cases (WHO 2003). Improved training is greatly needed, as is making available necessary equipment and supplies at all levels within health facilities.

Postabortion contraceptive service provision should be improved and coverage expanded to reduce women's risk for subsequent unintended pregnancies. Further improvements are needed in policies and programs to continue the positive trend toward increasing contraceptive use and to meet the growing demand for contraceptives. Additional research on the characteristics of women in Rwanda who obtain induced abortions would point to the populations in greatest need of interventions to prevent unintended pregnancy.

Programs to educate women and couples about the specifics of the abortion law (the criteria under which abortion is permitted) and how to obtain a legal procedure are needed, because most Rwandans believe abortion is illegal under all circumstances. Even though abortion can be legally accessed to save the life of the woman or to protect her physical health, and its cost is covered under Mutuelle, the current legal guidelines are so difficult to fulfill that even when legally allowed, safe abortion is likely beyond the reach of most women. In light of these findings, government officials should consider improving access to legal abortion by, for example, requiring that only one medical doctor determine the need for an abortion. Guidelines are also needed to clarify which types of health professionals and facilities are expected to provide this service. Though relatively small, such incremental changes can improve access to legal and safe abortion services. Rwanda remains a traditional and religiously conservative country. Even women's associations are divided about what should be done. Some cite cultural and religious beliefs as reasons to oppose abortion law reform. The estimates presented here of the incidence of abortion in Rwanda should provide a firm base of evidence for an open discussion of the level and negative impact of unintended pregnancy and unsafe abortion, and lead to the development of policies and programs to reduce them and strategies to protect the well-being of women and their families.

\section{Notes}

1 The 2007-08 Interim Demographic and Health Survey includes data from two weeks in 2007 but almost all data collection took place in 2008; therefore, we consider 2008 the year of the survey data and, for simplicity, we refer to the survey as the 2008 DHS.

2 Agrée health clinics are registered and receive assistance from the government but are operated by religious or nonprofit groups. Agrée clinics follow standard Ministry of Health guidelines and protocols. Some facilities with religious affiliations do not provide contraceptive services but have arrangements with, and refer clients to, public facilities that provide family planning services.

3 We asked for estimates of both types of pregnancy loss combined because facilities in legally restricted settings cannot readily differentiate the two types.

4 District health supervisors visit health centers in their districts on a quarterly basis and provide technical support and supervision.

5 In the Rwandan health system, healthcare providers are classified according to level of training and experience. A2 nurses, the majority of the health-care workforce, have completed secondary education in nursing school. A1 nurses are more highly trained and have completed high school and three additional years of training in nursing school.

6 The figure of 3.41 percent of all live births is arrived at through the following calculation. According to clinical studies of pregnancy loss by gestation (Harlap et al. 1980; Bongaarts and Potter 1983), miscarriages during 13-21 weeks gestation account for 2.9 percent of all recognized pregnancies in which abortion is not induced. Of all recognized pregnancies, 15 percent are estimated to be miscarriages (spontaneous pregnancy loss up to the twenty-first week of gestation) and 85 percent are live births. Thus, to calculate secondtrimester miscarriages as a proportion of the number of live births, 
we divided 2.9 percent by 85 percent, yielding 3.41 percent. Although some women who miscarry at earlier gestations seek medical care, those who seek treatment are likely to do so on an outpatient basis and relatively few are treated in facilities. Pregnancy losses at 22 or more weeks are not included because they are usually classified as fetal deaths rather than miscarriages.

7 The number of miscarriages was estimated using an accepted formula (10 percent of induced abortions and 20 percent of live births). For unintended pregnancies, the number of births would be those that are mistimed or unwanted, and for intended pregnancies the number of births would be those that are wanted at the time they occurred.

8 This is a different division of modern versus traditional methods than that used by DHS in their country reports in which the standard days method and lactational amenorrhea method are classified as modern methods. We classify these two methods as traditional because their failure is generally similar to that of other types of periodic abstinence methods.

\section{References}

Basinga, Paulin, Paul J. Gertler, Agnes Binagwaho, Agnes L. Soucat, Jennifer Sturdy, and Christel M. Vermeersch. 2011. "Effect on maternal and child health services in Rwanda of payment to primary health-care providers for performance: An impact evaluation," Lancet 377(9775): 1421-1428.

Bongaarts, John and Robert G. Potter (eds.). 1983. Fertility, Biology and Behavior: An Analysis of the Proximate Determinants. New York: Academic Press.

Government of Rwanda. 1980. "Codes et lois du Rwanda: Decret-Loi N²1/77 Code Penal, 18 août 1977."

Harlap, S., P. H. Shiono, and S. Ramcharan. 1980. "A life table of spontaneous abortions and the effects of age, parity and other variables," in Ian H. Porter and Ernest B. Hook (eds.), Human Embryonic and Fetal Death. New York: Academic Press, pp. 145-158.

Kanyesigye, Frank. 2011. “Clinic closed over abortion.” The New Times, 23 July.

Kwizera, Charles. 2011. "Long sentences for abortion counterproductive-Karugarama." The New Times, 4 May.

Levandowski, Brooke, Edgar Kuchingale, Linda Kalilani-Phiri, et al. 2011. "The estimated incidence of abortion in Malawi." Paper presented at the Annual Meeting of the Population Association of America, Washington, DC, 31 March.

Ministry of Finance and Economic Planning [Rwanda]. 2000. Rwanda Vision 2020. Kigali, Rwanda.

- 2002. A Profile of Poverty in Rwanda: An Analysis Based on the Results of the Household Living Condition Survey 1999-2001. Kigali, Rwanda: National Poverty Reduction Programme \& Statistics Department.

- 2007. Economic Development and Poverty Reduction Strategy, 20082012. Kigali, Rwanda: Ministry of Finance and Economic Planning.

Ministry of Health (MOH) [Rwanda], National Institute of Statistics of Rwanda (NISR), and ICF Macro. 2009. Rwanda Interim Demographic and Health Survey 2007-08. Calverton, MD: MOH, NISR, and ICF Macro.

National Institute of Statistics of Rwanda (NISR). 2006. Preliminary Poverty Update Report: Integrated Living Conditions Survey 2005-06 [Enquête Intégrale sur les Conditions de Vie des Ménages]. First draft for Consultation. Kigali, Rwanda.

2009. National Population Projection 2007-2022. Kigali, Rwanda.
National Institute of Statistics of Rwanda (NISR), Ministry of Health (MOH) [Rwanda], and ICF International. 2011. Rwanda Demographic and Health Survey 2010. Calverton, MD: NISR, MOH [Rwanda], and ICF International.

National Institute of Statistics of Rwanda (NISR) and ORC Macro. 2006. Rwanda Demographic and Health Survey 2005. Calverton, MD: NISR and ORC Macro.

Office National de la Population (ONP) and ORC Macro. 2001. Enquête Démographique et de Santé, Rwanda 2000. Kigali, Rwanda, and Calverton, MD: ONP and ORC Macro.

Pearson, L. and R. Shoo. 2005. "Availability and use of emergency obstetric services: Kenya, Rwanda, Southern Sudan, and Uganda," International Journal of Gynecology and Obstetrics 88(2): 208-215.

Rusa, Louis, Miriam Schneidman, Gyuri Fritsche, and Laurent Musango. 2009. "Rwanda: Performance-based financing in the public sector," in R. Eichler, R. Levine, and the Performace-Based Incentives Working Group (eds.), Performance Incentives for Global Health: Potential and Pitfalls. Washington, DC: Center for Global Development, pp. 189-214.

Singh, Susheela, Tamara Fetters, Hailemichael Gebreselassie, et al. 2010. "The estimated incidence of induced abortion in Ethiopia, 2008," International Family Planning Perspectives 36(1): 16-25.

Singh, Susheela, Elena Prada, and Fatima Juarez. 2011. “Incidence complications method: A quantitative technique," in Susheela Singh, Lisa Remez, and Alyssa Tartaglione (eds.), Methodologies for Estimating Abortion Incidence and Abortion-Related Morbidity: A Review. New York: Guttmacher Institute, pp. 71-99.

Singh, Susheela, Elena Prada, Florence Mirembe, and Charles Kiggundu. 2005. "The incidence of induced abortion in Uganda," International Family Planning Perspectives 31(4): 183-191.

Treatment and Research AIDS Center (TRAC). 2010. Knowledge and Behavior Related to HIV/AIDS Among Youth 15-24, Rwanda, 2009. Kigali.

United Nations (UN). 2011. “Goal 5: Improve Maternal Health.” <http:// www.un.org/millenniumgoals/maternal.shtml>. Accessed 4 May 2009.

Vyankandondera, Joseph. 2009. Personal communication with senior consultant, Obstetrical and Gynecology Department, Centre Hospitalier Universitaire de Kigali.

World Health Organization (WHO). 2003. Managing Complications in Pregnancy and Childbirth: A Guide for Midwives and Doctors. Geneva.

2011. Unsafe Abortion: Global and Regional Estimates of the Incidence of Unsafe Abortion and Associated Mortality in 2008. Geneva.

\section{Acknowledgments}

Research for this project was funded by the William and Flora Hewlett Foundation, the David and Lucille Packard Foundation, and the Government of the Netherlands. The authors are grateful to Laetitia Nyirazinyoye for comments on the study design, participation in data collection, and comments on an earlier draft of the article, and to Cyprien Munyanshongore for comments on the study design and participation in data collection. We thank Jean d'Amour Habagusenga and Nathalie Murindahabi for data management, and Suzette Audam for support with data processing. We are also thankful to members of the project's Advisory Panel and members of the Ministry of Health Maternal and Child Health Technical Working Group for their guidance and valuable comments on the protocol and study tools. We are grateful to Akinrinola Bankole, Lisa Remez, and Florina I. Serbanescu for their insightful comments on earlier drafts of this article. 